\title{
Chidamide induces apoptosis in DLBCL cells by suppressing the HDACs/STAT3/Bcl-2 pathway
}

\author{
HONGWEI ZHANG $^{1 *}$, FENQING CHI $^{2 *}$, KERU QIN $^{2}$, XIULI MU $^{2}$, LIEYANG WANG $^{1}$, BIN YANG $^{1}$, \\ YANLI WANG $^{1}$, MIN BAI ${ }^{1}$, ZHENHUA LI $^{1}$, LIPING SU$^{1}$ and BAOFENG YU ${ }^{2}$ \\ ${ }^{1}$ Department of Hematology, Cancer Hospital of Shanxi Province, Taiyuan, Shanxi 030013; \\ ${ }^{2}$ Department of Biochemistry and Molecular Biology, Key Laboratory of Cellular Physiology of \\ Ministry of Education, Shanxi Medical University, Taiyuan, Shanxi 030001, P.R. China
}

Received September 21, 2020; Accepted February 11, 2021

DOI: $10.3892 / \mathrm{mmr} .2021 .11947$

\begin{abstract}
Diffuse large B-cell lymphoma (DLBCL) is a highly heterogeneous malignant tumor type, and epigenetic modifications such as acetylation or deacetylation serve vital roles in its development. Chidamide, a novel histone deacetylase inhibitor, exerts an anticancer effect against various types of cancer. The present study aimed to evaluate the cellular effect of chidamide on a number of DLBCL cell lines and to investigate its underlying mechanism. The results demonstrated that chidamide induced the death of these cells in a concentration-(0-30 $\mu \mathrm{mol} / \mathrm{l})$ and time-dependent (24-72 h) manner, as determined using the Cell Counting Kit- 8 cell viability assay. Moreover, chidamide promoted cellular apoptosis, which was identified via flow cytometry and western blot analysis, with an increase in cleaved caspase- 3 expression and a decrease in Bcl-2 expression. Chidamide treatment also decreased the expression level of STAT3 and its phosphorylation, which was accompanied by the downregulation of a class-I histone deacetylase (HDAC) inhibitor, chidamide. Collectively, these data suggested that chidamide can be a potent therapeutic agent to treat DLBCL by inducing the apoptotic death of DLBCL cells by inhibiting the HDACs/STAT3/Bcl-2 pathway.
\end{abstract}

Correspondence to: Professor Liping $\mathrm{Su}$, Department of Hematology, Cancer Hospital of Shanxi Province, 3 Zhigongxinjie Street, Xiehualing, Taiyuan, Shanxi 030013, P.R. China

E-mail: sulp2005@sohu.com

Professor Baofeng Yu, Department of Biochemistry and Molecular Biology, Key Laboratory of Cellular Physiology of Ministry of Education, Shanxi Medical University, 56 Xinjiannan Road, Taiyuan, Shanxi 030001, P.R. China

E-mail: shanxiyangcheng@126.com

${ }^{*}$ Contributed equally

Abbreviations: DLBCL, diffuse large B-cell lymphoma; CCK-8, Cell Counting Kit-8; HDAC, histone deacetylase

Key words: DLBCL, chidamide, HDAC, STAT3, Bcl-2, apoptosis

\section{Introduction}

Diffuse large B-cell lymphoma (DLBCL) is a genetically and clinically heterogeneous lymphoid malignancy type, which represents the most common category and disease entity of non-Hodgkin lymphoma in adults and accounts for $\sim 30-40 \%$ of all cases in different geographic regions, such as Europe and the USA $(1,2)$. In total, two major molecular subtypes of DLBCL have been identified via gene expression profiling: Germinal center B cell-like (GCB) and activated B cell-like (ABC), which represent lymphomas derived from different stages of B-cell lymphoid differentiation (3). GCB- and ABC-subtype DLBCLs have different oncogenic pathways but they share similar morphologies such as a white, 'fish-flesh', homogeneous cut surface (4). Thus far, the use of the R-CHOP regimen (including rituximab, cyclophosphamide, doxorubicin, vincristine and prednisone) as a standard component of immunochemotherapy has greatly improved the prognosis of patients with DLBCL, with a response rate of $\sim 80 \%$ (5). However, some patients with DLBCL do not respond to the R-CHOP treatment due to disease heterogeneity (6). Thus, alternative or supplemental strategies are required for these subsets of patients with DLBCL.

STAT3 is a transcription activation factor, and participates in the malignant progression of multiple tumors, including DLBCL (7). RNA in situ hybridization (RNA scope) has revealed that there are more STAT3 positive cells in the ABC-subtype of DLBCL tissue samples compared with those of the GCB-subtype of DLBCL tissue samples (8). Activation or phosphorylation of STAT3 is strongly associated with more advanced clinical stage and overall poor survival of patients with DLBCL (9). Therefore, inhibition of STAT3 activation provides an additional therapeutic target for DLBCL, especially for ABC-subtype DLBCL (10).

Chidamide is a benzamide histone deacetylase (HDAC) inhibitor, and it has been marketed in China for the treatment of peripheral T-cell lymphoma (PTCL) since 2015 (11). Chidamide has also been used to treat myeloma, non-small lung cancer types and breast cancer due to its antitumor potency (12-14). Although the antitumor mechanism of chidamide has been widely investigated, studies examining 
the target profiling for chidamide are sparse, which limits its further application in the treatment of multiple tumors.

To provide evidence for the clinical application of chidamide in DLBCL, the present study examined the cytotoxic effect of chidamide on DLBCL cells and its possible underlying molecular mechanism.

\section{Materials and methods}

Cells and cell culture. Human DLBCL cell lines SU-DHL2 and MZ were purchased from Jennio-Bio, Co., Ltd., and the OCI-LY3 cell line was from Beina Chuanglian Biological Research Institute (Beijing, China). SU-DHL2 and OCI-LY3 cells of the ABC-subtype, and the MZ cells of the GCB-subtype of DLBCL cells were cultured in RPMI-1640 medium (Gibco; Thermo Fisher Scientific, Inc.) containing 10\% FBS (Gibco; Thermo Fisher Scientific, Inc.) and 1\% penicillin-streptomycin (Gibco; Thermo Fisher Scientific, Inc.). All cells were cultured in a $37^{\circ} \mathrm{C}$ incubator with $5 \% \mathrm{CO}_{2}$. All three cell lines were tested for mycoplasma contamination and authenticated by STR profiling.

Reagents and antibodies. Chidamide was purchased from Absin Bioscience, Inc., and dissolved in DMSO (Sigma-Aldrich; Merck KGaA) to prepare the stock solution (30 mM), which was stored at $-20^{\circ} \mathrm{C}$ in the dark. A Cell Counting Kit-8 (CCK-8) was obtained from Beijing Solarbio Biotechnology Co., Ltd. The Annexin V-FITC/PI kit, which was used for apoptotic cell death determination via flow cytometry, was purchased from Beyotime Institute of Biotechnology. Antibodies against cleaved caspase-3 (cat. no. 9664S; 1:1,000), Bcl-2 (cat. no. 15071S; 1:1,000), HDAC1 (cat. no. 34589S; 1:1,000), HDAC2 (cat. no. 57156S; 1:1,000), HDAC3 (cat. no. 85057S; 1:1,000), STAT3 (cat. no. 9139S; 1:1,000), phosphorylated (P)-STAT3-705 (cat. no. 9145S; 1:1,000), P-STAT3-727 (cat. no. 49081S; $1: 1,000$ ) and $\beta$-actin (cat. no. 3700S; 1:5,000) were purchased from Cell Signaling Technology, Inc. An antibody against HDAC8 (cat. no. JJ0845; 1:1,000) was purchased from Novus Biologicals, LLC.

Cell viability assay. SU-DHL2, OCI-LY3 and MZ DLBCLcells $\left(2 \times 10^{5}\right.$ cells $\left./ \mathrm{ml}\right)$ were seeded into 96 -well plates at a volume of $100 \mu \mathrm{l} /$ well and then treated with $5 \mu \mathrm{l} /$ well chidamide at different final concentrations $(0,0.1,0.3,1,3,10$ and $30 \mu \mathrm{M})$ for 24,48 and $72 \mathrm{~h}$ in a $37^{\circ} \mathrm{C}$ incubator with $5 \% \mathrm{CO}_{2}$, respectively, to examine the concentration- and time-dependence of cellular chidamide effects. Equal molar concentrations of DMSO were used in the control group. At the indicated time of the reaction, $10 \mu \mathrm{l} \mathrm{CCK}-8$ reagent was added to each well and incubated at $37^{\circ} \mathrm{C}$ for $2 \mathrm{~h}$, according to the manufacturer's instructions. The final absorbance [optical density (OD) value] of the cultured cells was measured at a wavelength of $450 \mathrm{~nm}$ with a SynergyHTX microplate reader (BioTek Instruments, Inc.). Cell viability was calculated and expressed as the ratio of the OD450 values for chidamide-treated cells over those of the DMSO-treated control cells. The drug concentration and cell survival rate were entered into GraphPad Prism software 5.0 (GraphPad Software, Inc.) to obtain a concentration-survival rate curve. The half maximal inhibitory concentration $\left(\mathrm{IC}_{50}\right)$, which represents the concentration of an inhibitor (drug) that is required for $50 \%$ inhibition of cells (15), was calculated. The concentration corresponding to the point where this line and the curve intersect is the $\mathrm{IC}_{50}$. The specific value is directly obtained using the software and displayed in the results.

Flow cytometry analysis. Cells were seeded in 6-well flat-bottomed plates at a density of $3 \times 10^{5}$ cells/well and treated with chidamide at different concentrations $(0,1,2.5$, 5 and $10 \mu \mathrm{M}$ ) in a $37^{\circ} \mathrm{C}$ incubator with $5 \% \mathrm{CO}_{2}$ for $48 \mathrm{~h}$. The cells were then collected, and their density was adjusted to $5 \times 10^{5}$ cells $/ \mathrm{ml}$. Then, $100 \mu \mathrm{l}$ cell suspension was incubated with $5 \mu \mathrm{l}$ Annexin V-FITC at room temperature for $10 \mathrm{~min}$ in the dark, followed by centrifugation at $1,000 \mathrm{x}$ for $5 \mathrm{~min}$ at room temperature. Subsequently, $10 \mu \mathrm{l}$ PI staining solution was added to the Annexin V-FITC stained cells for $15 \mathrm{~min}$ at room temperature in dark, which were then analyzed via flow cytometry (FACSCanto II; BD Biosciences) and FACSCanto Clinical Software (v3.0; BD Biosciences) to determine the percentage of Annexin $\mathrm{V}^{+} / \mathrm{PI}^{-}$(early apoptotic) and Annexin $\mathrm{V}^{+} / \mathrm{PI}^{+}$(late apoptotic) cells. The Annexin V-FITC apoptotic cell detection kit (Beijing TransGen Biotech Co., Ltd.) used included PI to label the cellular DNA of dead cells with a compromised total membrane. Thus, the kit allows the differentiation among early apoptotic cells (Annexin $\mathrm{V}^{+}, \mathrm{PI}^{-}$), late apoptotic cells (Annexin $\mathrm{V}^{+}, \mathrm{PI}^{+}$) and viable cells (Annexin $\mathrm{V}^{-}, \mathrm{PI}^{-}$) in chidamide-treated DLBCL cells. Early and late apoptosis were analyzed, and the apoptotic rate was calculated as the percentage of early and late apoptotic cells.

$R N A$ extraction and reverse transcription-quantitative (RT- $q)$ PCR analysis. SU-DHL2, OCI-LY3 and MZ DLBCL cells were treated for $48 \mathrm{~h}$ with chidamide $(0,2.5,5$ and $10 \mu \mathrm{M})$ in a $37^{\circ} \mathrm{C}$ incubator with $5 \% \mathrm{CO}_{2}$, and total RNAs were extracted from the cells using TRIzol ${ }^{\circledR}$ reagent (Invitrogen; Thermo Fisher Scientific, Inc.) according to the manufacturer's protocols. RT-qPCR was performed with a UltraSYBR One Step RT-qPCR kit (cat. no. CW0659S; CWBio) using the ABI 7500 Real-time PCR system (Applied Biosystems; Thermo Fisher Scientific, Inc.) in duplicate for each reaction. The reverse transcription of total RNA into cDNA was performed at $45^{\circ} \mathrm{C}$ for $10 \mathrm{~min}$. The following thermocycling conditions were used for qPCR: Initial denaturation at $95^{\circ} \mathrm{C}$ for $5 \mathrm{~min}$; followed by 30 cycles at $95^{\circ} \mathrm{C}$ for $10 \mathrm{sec}$ and $60^{\circ} \mathrm{C}$ for $45 \mathrm{sec}$. Averages of the obtained $\mathrm{Cq}$ values were used for further calculations. Gene expression levels were normalized to the expression of the endogenous control gene, $\beta$-actin. The gene expression levels were calculated using the $2^{-\Delta \Delta \mathrm{Cq}}$ method (16). The primer sequences used for the qPCR were as follows: STAT3 forward, 5'-GGAGGAGTTGCAGCAAAAAG-3' and reverse, 5'-TGT GTTTGTGCCCAGAATGT-3'; and $\beta$-actin forward, 5'-CTG GCACCACACCTTCTACA-3' and reverse, 5'-AGCACAGCC TGGATAGCAAC-3'.

Western blot analysis. After SU-DHL2, OCI-LY3 and MZ DLBCL cells were treated for $48 \mathrm{~h}$ with chidamide (at $0,2.5$, 5 and $10 \mu \mathrm{M}$ ) in a $37^{\circ} \mathrm{C}$ incubator with $5 \% \mathrm{CO}_{2}$, proteins were extracted from these cells using RIPA lysis buffer (Beyotime Institute of Biotechnology) and quantified using the BCA protein assay reagent (CWBio). SDS-PAGE with $10 \%$ gels was used to isolate proteins (30 $\mu \mathrm{g}$ per lane). The proteins were 

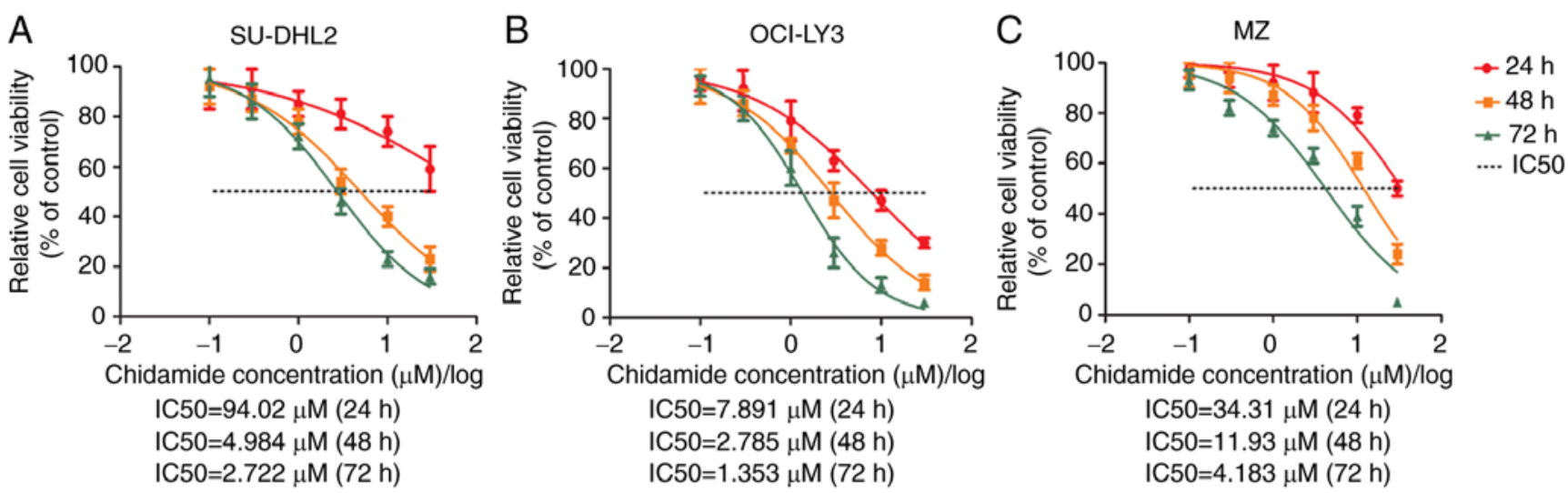

Figure 1. Chidamide inhibits diffuse large B-cell lymphoma cell viability. (A) SU-DHL2, (B) OCI-LY3 and (C) MZ cells were treated with chidamide at the indicated concentrations for 24,48 , and $72 \mathrm{~h}$. Cell viability was determined and the $\mathrm{IC}_{50}$ was calculated accordingly. The experiments were repeated three times. Data are presented as the mean $\pm \mathrm{SD}$ from three independent experiments performed in triplicate.

transferred to PVDF membranes, which were then blocked with $5 \%$ BSA (Sigma-Aldrich; Merck KGaA) for $1 \mathrm{~h}$ at room temperature. After incubation with the primary antibodies overnight at $4^{\circ} \mathrm{C}$, the membranes were washed three times with Tween-20 (1:1,000 dilution)-PBS for $10 \mathrm{~min}$ each. Finally, the membranes were treated with the appropriate HRP-conjugated secondary antibodies (cat. nos. CW0103S and CW0102S; 1:5,000; CWBio) for $1 \mathrm{~h}$ at room temperature, and protein bands were visualized with ECL reagents (Corning, Inc.). Images were captured and analyzed with a Universal Hood II Chemiluminescence Imaging system (Bio-Rad Laboratories, Inc.). Densitometric analysis was performed on the instrument.

Statistical analysis. Data are presented as the mean \pm SD for the number of indicated replicates. Statistical analyses were conducted using GraphPad Prism version 5.0 (GraphPad Software, Inc.). A Student's t-test was used to compare the difference between two groups. Homogeneity of variance was assessed using Brown-Forsythe test. Comparisons among multiple groups were performed using one-way ANOVA followed by Tukey's test. $\mathrm{P}<0.05$ was considered to indicate a statistically significant difference.

\section{Results}

Chidamide inhibits the viability of DLBCL cells. To investigate the cellular effect of chidamide on DLBCL cells, three cell lines of SU-DHL2, OCI-LY3 and MZ were selected for this study. These cells were treated with chidamide at different final concentrations $(0,0.1,0.3,1,3,10$ and $30 \mu \mathrm{M})$ for 24,48 and $72 \mathrm{~h}$. As presented in Fig. 1, the exposure of these DLBCL cells to chidamide reduced their viability in both concentration- and time-dependent manners, as determined via the cellular CCK-8 assay. For instance, after $72 \mathrm{~h}$ of treatment, the calculated $\mathrm{IC}_{50}$ values for the concentration-dependent effect of chidamide inhibition were $2.722 \mu \mathrm{M}$ for SU-DHL20 cells, $1.353 \mu \mathrm{M}$ for OCI-LY3 cells and $4.183 \mu \mathrm{M}$ for MZ cells (Fig. 1). Studies of the time dependence of cell viability by chidamide indicated that, for SU-DHL20 cells, the $\mathrm{IC}_{50}$ values were $94.02 \mu \mathrm{M}$ at $24 \mathrm{~h}, 4.984 \mu \mathrm{M}$ at $48 \mathrm{~h}$ and $2.722 \mu \mathrm{M}$ at
$72 \mathrm{~h}$ (Fig. 1A). The $\mathrm{IC}_{50}$ decreased with increasing chidamide concentration and treatment time in these DLBCL cell lines. These data suggested that chidamide can inhibit or decrease the cell viabilities of these three DLBCL cell lines with different potency.

Chidamide induces apoptosis in DLBCL cells. As the malignancy of DLBCL cells has been associated with the inhibition of apoptosis (17), it was next determined whether apoptosis was associated with the chidamide-induced reduction of the DLBCL cell viability. SU-DHL2, OCI-LY3 and $\mathrm{MZ}$ cells were treated with chidamide at different concentrations $(0,1,2.5,5$ and $10 \mu \mathrm{M})$ for $48 \mathrm{~h}$, and the apoptotic nature of cell death was determined via flow cytometry assays and western blot analysis of apoptosis-related proteins. As presented in Fig. 2, quantitative flow cytometry indicated that Annexin V-FITC staining of apoptotic cells was evident in all the three types of chidamide-treated DLBCL cells in a dose-dependent manner (Fig. 2). Early apoptotic cells were consistently present in all three types of chidamide-treated DLBCL cells, and the percentages of late apoptotic cells were increased as the chidamide concentration was increased after $48 \mathrm{~h}$ exposure (Fig. 2). Moreover, flow cytometry assays demonstrated that SU-DHL2 cells were more sensitive to chidamide.

Western blot analysis of total proteins extracted from the cells exposed to chidamide revealed an enhanced expression level of cleaved caspase- 3 and a decreased expression level of Bcl-2 (Fig. 3), a further hallmark of apoptosis (18); these effects were exerted in a concentration-dependent manner. These results suggest that chidamide inhibits cell viability in all three types of DLBCL cells by stimulating a mechanism of apoptotic cell death.

Chidamide suppresses the expression levels of class I HDACs. Chidamide is a selective and potent inhibitor of the activities of class I HDAC enzymes, including HDAC1, HDAC2, HDAC3 and HDAC8 (19). To investigate the possible mechanism of chidamide-evoked apoptosis in DLBCL cells, the expression levels of these class I HDACs were evaluated via semi-quantitative western blot analysis in chidamide-treated 

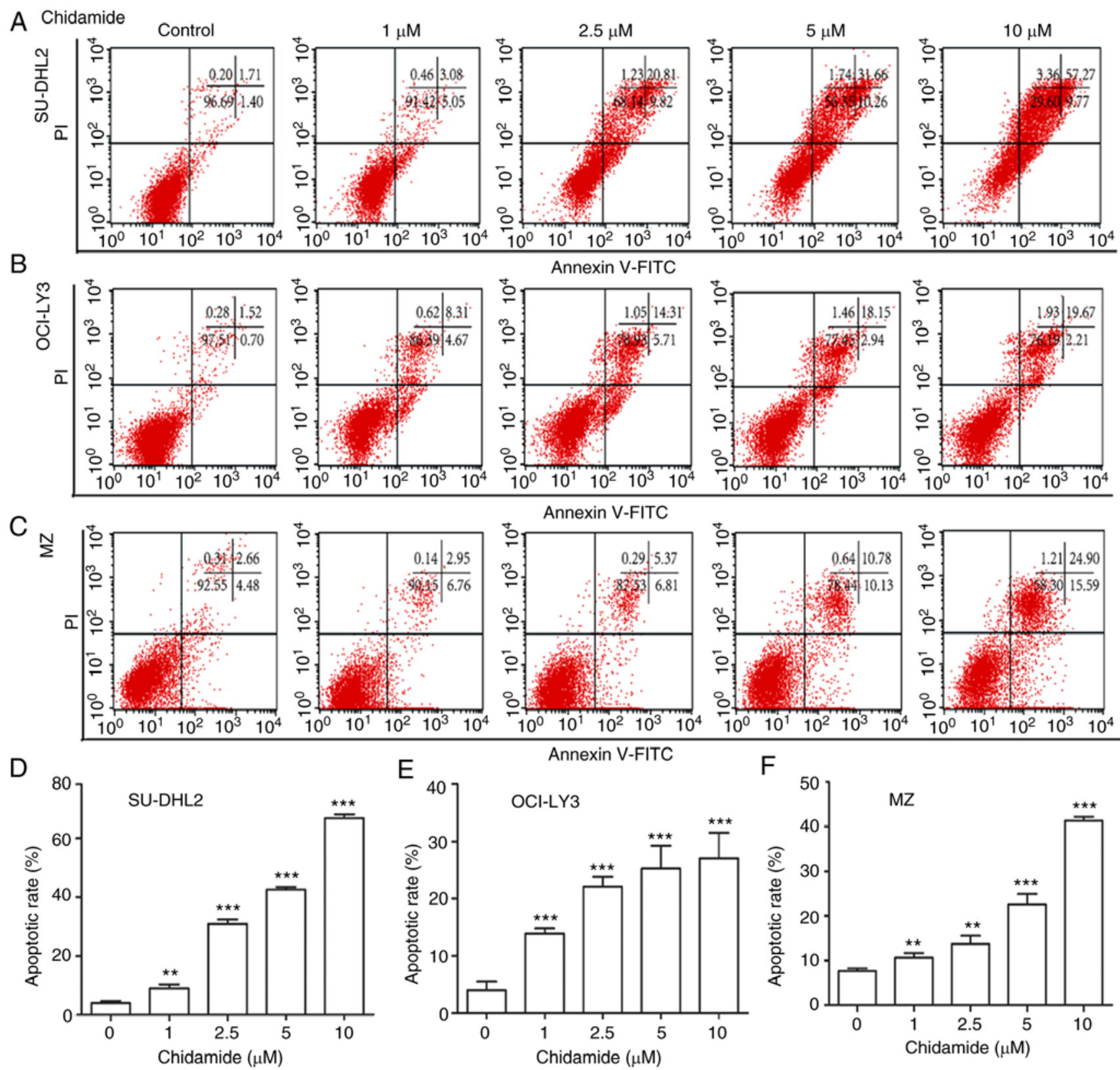

Figure 2. Flow cytometry analysis of chidamide-induced apoptosis in DLBCL cells. (A) SU-DHL2, (B) OCI-LY3 and (C) MZ cells were treated with chidamide at the indicated concentrations for $48 \mathrm{~h}$, double stained with Annexin V-FITC and PI, and analyzed via quantitative flow cytometry assay. Quantification of apoptosis results for (D) SU-DHL2, (E) OCI-LY3 and (F) MZ cells. The experiments were repeated three times in triplicates for each. ${ }^{* *} \mathrm{P}<0.01$ and ${ }^{* * * *} \mathrm{P}<0.001$ vs. control group without chidamide.

SU-DHL2, OCI-LY3 and MZ cells. Chidamide administration significantly decreased the expression levels of HDAC1, HDAC2, HDAC 3 and HDAC8 in a concentration-dependent manner after $48 \mathrm{~h}$ (Fig. 4). These results demonstrate that chidamide can suppress the expression levels of class I HDAC enzymes in all the three DLBCL cell lines tested under the current experimental conditions.

Chidamide inhibits the activation of STAT3 in DLBCL cells. Activation or phosphorylation of STAT3 has been reported to serve a critical role in the malignant progression of multiple tumors, including DLBCL (7). To further examine the possible mechanism of chidamide-induced death of DLBCL cells, it was next investigated whether chidamide influences the expression levels of STAT3 and its phosphorylation in the DLBCL cells. The results demonstrated that chidamide exposure significantly decrease both the mRNA and protein expression levels of STAT3 in SU-DHL2, OCI-LY3 and MZ cells in a concentration-dependent manner during a $48 \mathrm{~h}$ treatment, as determined via RT-qPCR (Fig. 5) and western blotting (Fig. 6). The levels of STATs phosphorylation in these chidamide-treated DLBCL cells were determined via western blotting, and a concentration-dependent reduction of STAT3 phosphorylation was also identified in SU-DHL2, OCI-LY3 and MZ cells. Collectively, these results indicate that chidamide treatment suppresses STAT3 protein expression and also inhibits its activation by phosphorylation in the tested DLBCL cells. 
A

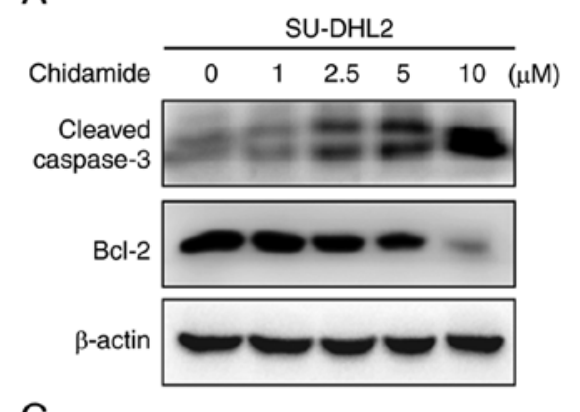

C

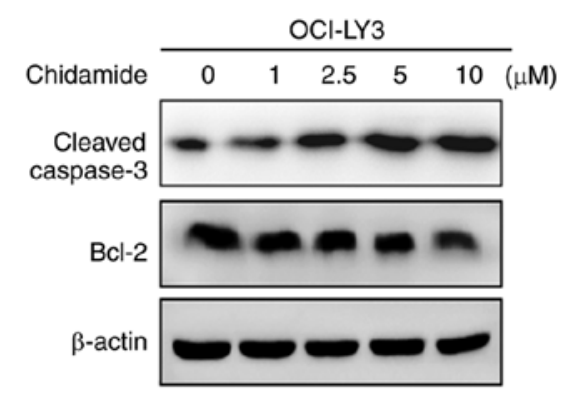

$\mathrm{E}$

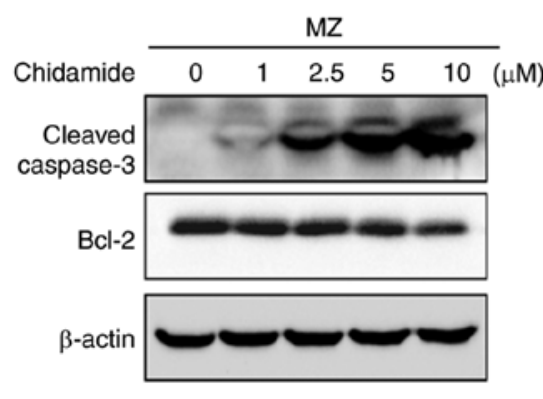

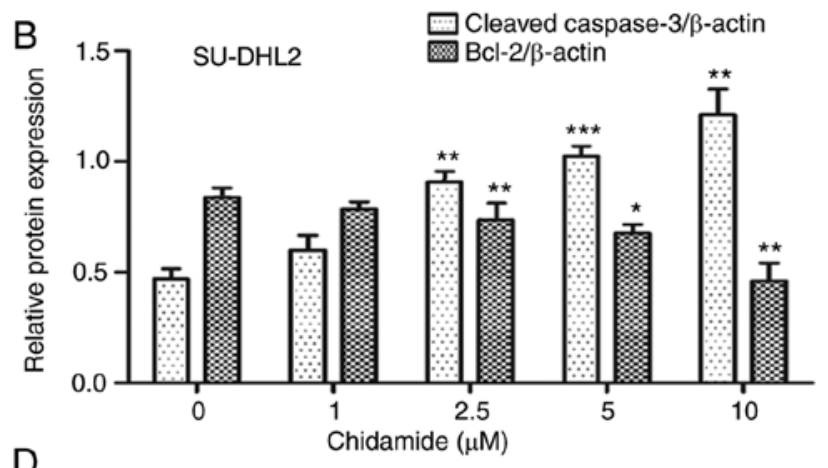

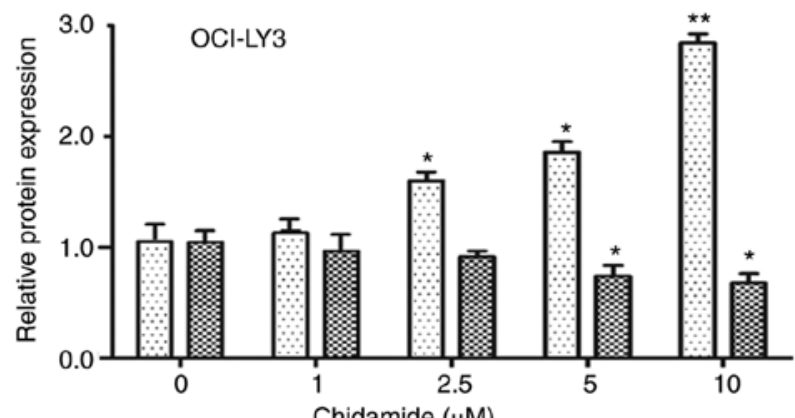

$\mathrm{F}$

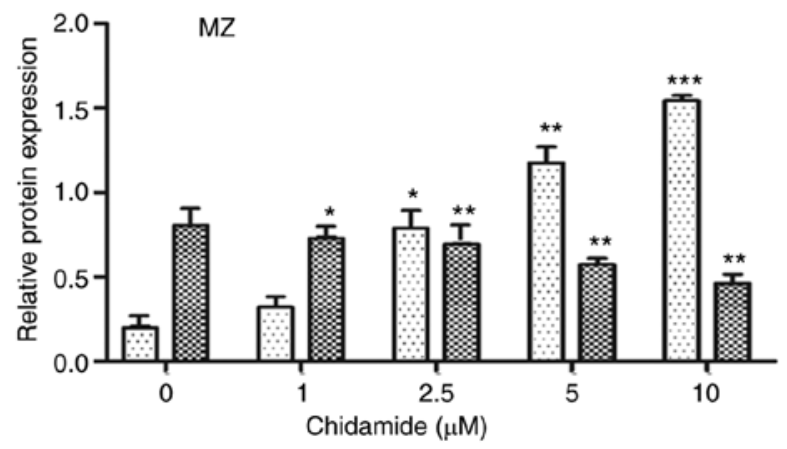

Figure 3. Western blot analysis of chidamide-induced apoptosis in DLBCL cells. (A and B) SU-DHL2, (C and D) OCI-LY3 and (E and F) MZ cells were treated with chidamide at the indicated concentrations for $48 \mathrm{~h}$, and western blotting was used to determine the expression levels of the apoptosis-related cleaved-caspase- 3 and $\mathrm{Bcl}-2$ proteins. $\beta$-actin served as the internal control. Data are present as the mean $\pm \mathrm{SD}$ from three sets of independent experiments. ${ }^{*} \mathrm{P}<0.05,{ }^{* *} \mathrm{P}<0.01$ and ${ }^{* * *} \mathrm{P}<0.001$ vs. control group without chidamide.

\section{Discussion}

It has been well documented that histone acetylation, which is regulated by the opposing activities of histone acetyltransferases and HDACs, serves a critical role in the development and progression of cancer by modulating gene transcription, chromatin remodeling and nuclear architecture (20). Along with histones, HDACs can also deacetylate numerous non-histone cellular substrates, such as the transcription factors p53 and NF- $\mathrm{B}$ (21), to regulate a wide variety of biological processes, including cancer initiation and progression (20). Therefore, HDAC inhibitors, acting as a potential therapeutic agent, are becoming increasing promising in cancer treatment.

A high level of HDAC expression has been well established in DLBCL cell lines and tissue sections, especially for class I HDACs that are closely associated with the survival of patients with DLBCL $(22,23)$. Accordingly, HDAC inhibitors have been widely used in clinical trials for DLBCL management (24). As a novel and orally active benzamide class of HDAC inhibitor, chidamide has thus been approved by the Chinese Food and Drug Administration authority in 2015 for the treatment of lymphoma, in particular for PTCL, since it can selectively inhibit the activities of class I HDACs, including HDAC2, 3 and 10 (25-27). Importantly, the present study has further demonstrated that chidamide can suppress the expression of HDAC1, 2, 3 and 8 of the class I HDACs in three different DLBCL cell lines in a concentration-dependent manner. These data suggest that chidamide regulates the functions of HDACs by influencing their abundance and activities, and thus enhances the current understanding of the role of chidamide in HDACs.

To evaluate the clinical potential of chidamide in the treatment of DLBCL, the current study determined whether chidamide affects the viability of three different DLBCL cell lines; SU-DHL2 and OCI-LY3 cells of the ABC-subtype and $\mathrm{MZ}$ cells of the GCB-subtype. The present study found that exposure of these DLBCL cells to chidamide for $72 \mathrm{~h}$ evoked both concentration- and time-dependent inhibition of cell viability, displaying $\mathrm{IC}_{50}$ values of $2.722 \mu \mathrm{M}$ for SU-DHL2 


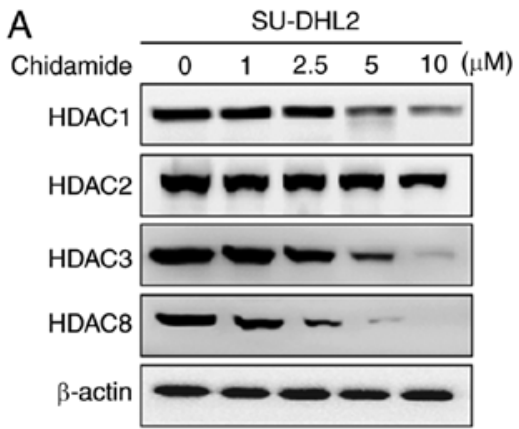

C

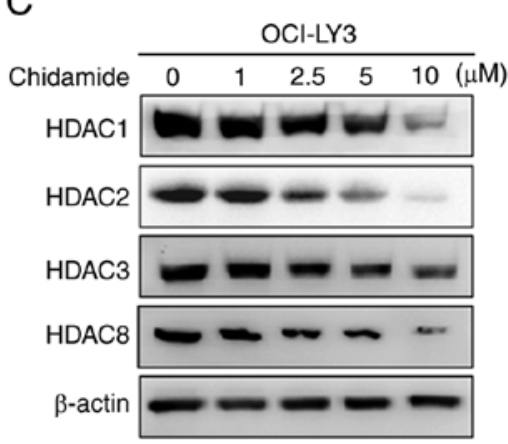

$\mathrm{E}$

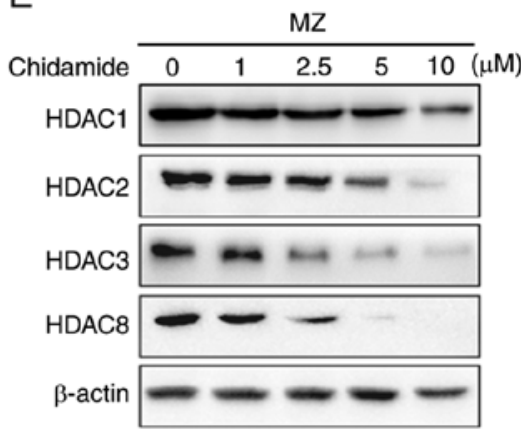

B

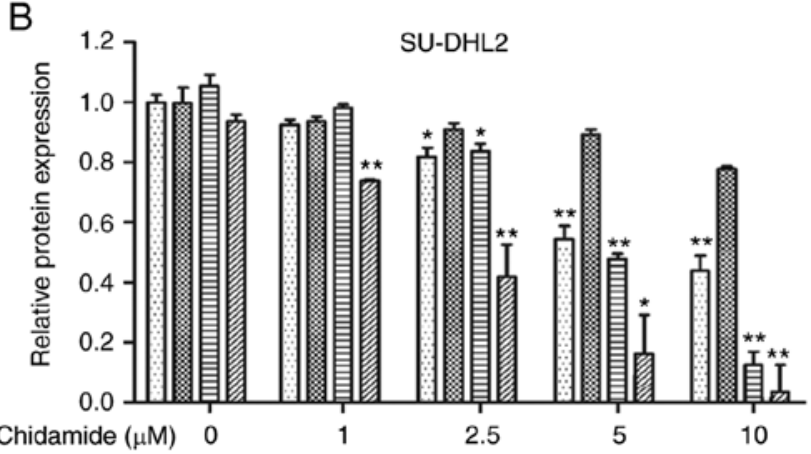

D

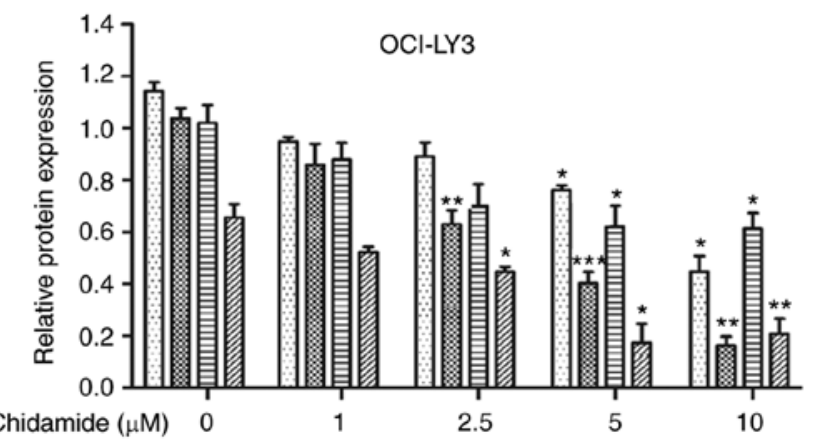

$\mathrm{F}$

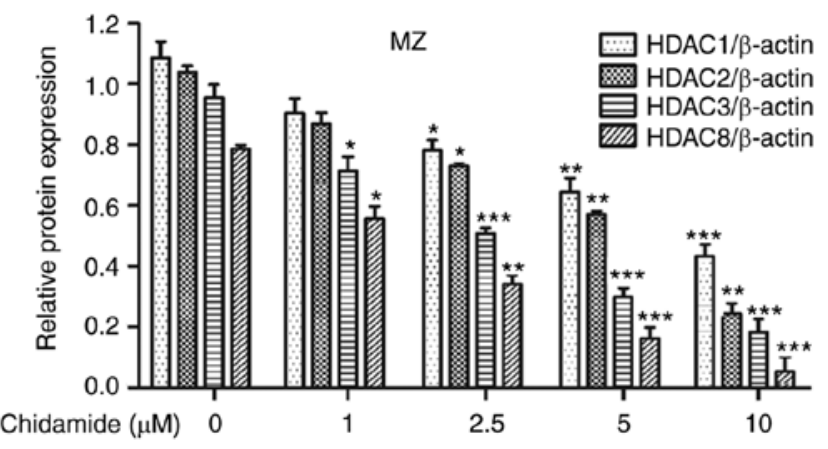

Figure 4. Chidamide decreases the expression of HDACs in DLBCL cells. (A and B) SU-DHL2, (C and D) OCI-LY3 and (E and F) MZ cells were treated with chidamide at concentrations from $0-10 \mu \mathrm{M}$ for $48 \mathrm{~h}$, and the expression levels of HDAC1, HDAC2, HDAC3 and HDAC8 were determined using western blotting. $\beta$-actin served as the internal control in all experiments. Data are presented as the mean $\pm \mathrm{SD}$ of three sets of experimental data. ${ }^{*} \mathrm{P}<0.05,{ }^{* * *} \mathrm{P}<0.01$ and ${ }^{* * *} \mathrm{P}<0.001$ vs. control group without chidamide. HDAC, histone deacetylase.

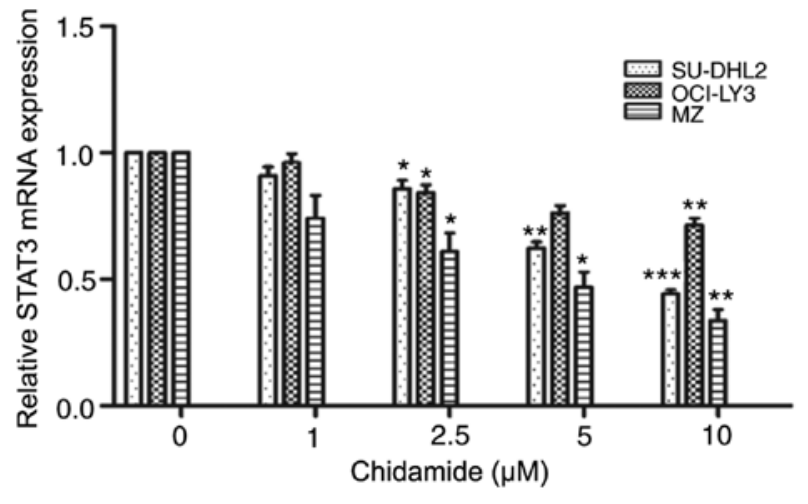

Figure 5. Chidamide represses the mRNA expression of STAT3 in DLBCL cells. SU-DHL2, OCI-LY3 and MZ cells were treated with chidamide at $0,1,2.5,5$ and $10 \mu \mathrm{M}$ for $48 \mathrm{~h}$. The mRNA expression level of STAT3 was assessed via reverse transcription-quantitative PCR, and results are expressed as ratios of $2^{-\Delta \Delta \mathrm{Cq}}$ values. All data are presented as the mean $\pm \mathrm{SD}$ of three sets of experimental data. ${ }^{*} \mathrm{P}<0.05,{ }^{* *} \mathrm{P}<0.01$ and ${ }^{* * *} \mathrm{P}<0.001$ vs. control group without chidamide. cells, $1.353 \mu \mathrm{M}$ for OCI-LY3 cells and $4.183 \mu \mathrm{M}$ for MZ cells. While these data do suggest that chidamide is a potent inhibitor of cell viability in DLBCL cells, they cannot provide conclusive information on which subtype of DLBCL cells is more sensitive to chidamide as only three cell lines were analyzed here.

To further examine the mechanism of chidamide-evoked inhibition of cell viability in DLBCL cells, flow cytometry and western blot analyses were performed to determine whether chidamide induces apoptosis in these DLBCL cells. There is evidence that apoptosis is involved in the initiation, progress and chemotherapy resistance of DLBCL (28), and that apoptosis can serve a vital role in governing the turnover of normal cells to transformed cells. The present flow cytometry analysis results of Annexin V-FITC staining of apoptotic cells identified that early apoptotic cells (Annexin $\mathrm{V}^{+}, \mathrm{PI}^{-}$) were consistently evident in all three types of chidamide-treated DLBCL cells, and that the percentages 
A

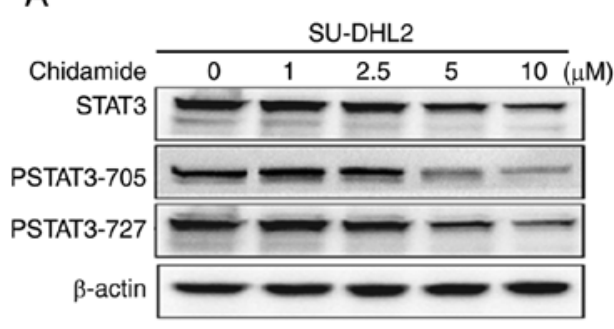

C

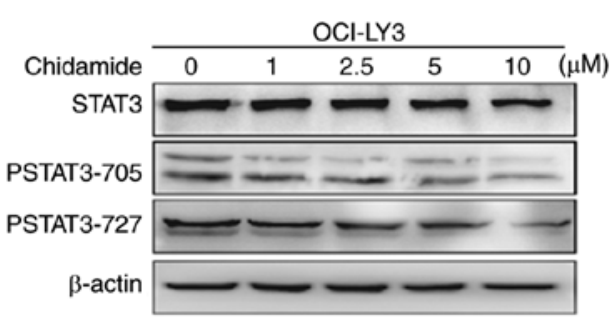

E

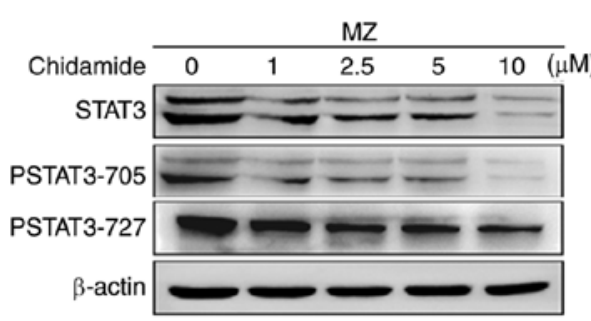

B

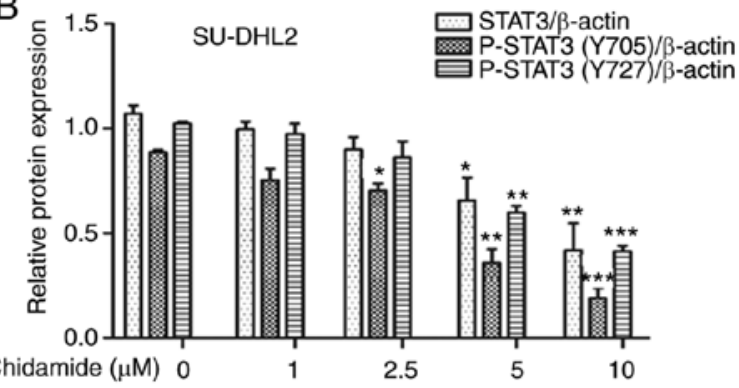

D

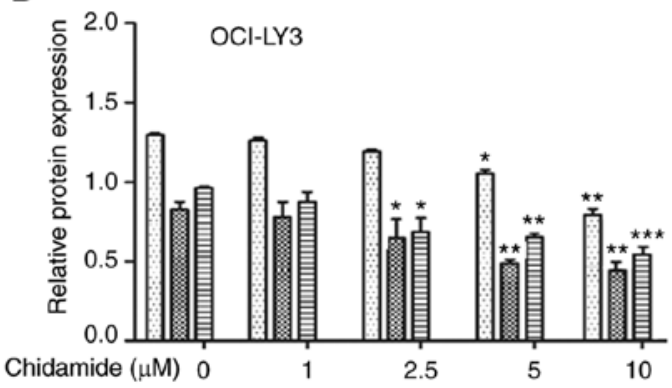

F

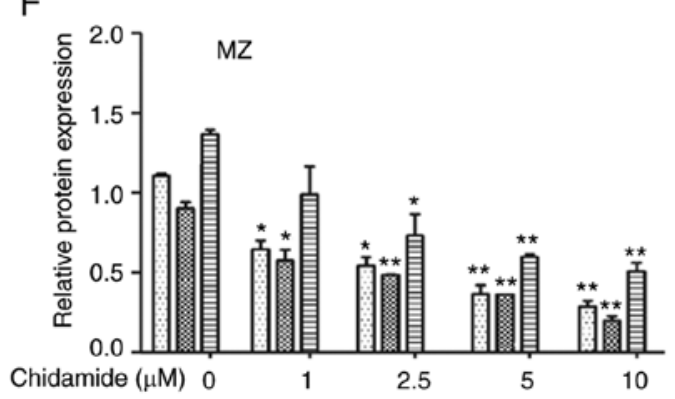

Figure 6. Chidamide inhibits the activation of STAT3 in DLBCL cells. (A and B) SU-DHL2, (C and D) OCI-LY3 and (E and F) MZ cells were treated with chidamide at $0,1,2.5,5$ or $10 \mu \mathrm{M}$ for $48 \mathrm{~h}$. The protein expression levels of STAT3 and its phosphorylation were measured via western blotting. $\beta$-actin served as the internal control in all experiments. Data are presented as the mean \pm SD of three sets of experimental data. ${ }^{*} \mathrm{P}<0.05,{ }^{* *} \mathrm{P}<0.01$ and ${ }^{* * * *} \mathrm{P}<0.001$ vs. control group without chidamide. P, phosphorylated.

of their late apoptotic cells $\left(\right.$ Annexin $\left.\mathrm{V}^{+}, \mathrm{PI}^{+}\right)$were increased in a chidamide concentration-dependent manner following $48 \mathrm{~h}$ exposure. Herein, flow cytometry assays demonstrated that SU-DHL2 cells were more sensitive to chidamide, possibly due to the heterogeneity of these DLBCL cells. It is well established that OCI-Ly3 cells contain the MYD88-L256P mutation, while SU-DHL2 cells possess the mutations of MYD88-S222R and TNFAIP3/A20 (29,30). The diverse mutation statuses of these cell lines may be the primary causes of their various sensitivities to chidamide treatment. Western blot analysis of total proteins extracted from these chidamide-exposed cells further revealed the apoptotic nature of cells, as indicated by enhanced cleavage of caspase- 3 and reduction of $\mathrm{Bcl}-2$ protein expression. These data support the previously reported pro-apoptotic effect of chidamide on DLBCL cells $(27,31)$. Taken together, these results suggest that inhibition of cell viability by chidamide is executed by stimulating apoptotic cell death in DLBCL cells.

As a transcription factor, STAT3 is constitutively activated in various cancer cell lines and tumor tissues, where it promotes tumor cell proliferation, invasion and migration (32).
The activity of STAT3 is regulated by diverse mechanisms, including an array of post-translational modifications, such as phosphorylation (e.g., Tyr705 and Ser727) (33). Since STAT3 serves vital roles in the progression of most human cancer types, it has been suggested that STAT3 could be an important candidate therapeutic target for tumor treatment (34). It has also been reported that different mechanisms are involved in STAT3 inhibition, including suppression of tyrosine enzymes that contribute to the phosphorylation or activation of STAT3, inhibition of STAT3-mediated transcription, repression of nuclear localization of STAT3 and inhibition of the interaction between STAT3 and its target gene (35). Nevertheless, remains unknown if and how chidamide influences STAT3 in DLBCL cells.

The present study demonstrated that the total mRNA and protein expression levels of STAT3 were progressively decreased by the administration of increasing concentrations of chidamide in these DLBCL cells. A concentration dependent suppression of STAT3 phosphorylation (Tyr705 and Ser727) was also induced by chidamide treatment in DLBCL cells. Interestingly, recent evidence has indicated that valproic acid, another HDAC inhibitor, can inhibit STAT3 
Tyr705 phosphorylation, but had no effect on the total protein expression level of STAT3 in natural killer cells (36). This difference with the current findings may be due to the fact that different HDAC inhibitors and cell types were used. The present results indicated that chidamide could regulate STAT3 activities via its protein expression, post-translational modification or both. It has been reported that p65 and p50 $\mathrm{NF}-\kappa \mathrm{B}$ interact physically with STAT3, thereby facilitating NF- $\kappa B$ recruitment to STAT3 promoters to modulate STAT3 expression (37). Thus, it was suggested that administration of chidamide may attenuate the transcriptional activity of $\mathrm{NF}-\kappa \mathrm{B}$ along with its inhibition of HDACs activities to suppress STAT3 transcription. Although the underlying mechanism via which chidamide restrains the expression of STAT3 requires further study, the present results also demonstrated that expression level of the anti-apoptotic Bcl-2 protein, a downstream target of STAT3 (38), was attenuated by chidamide treatment, which decreased the expression level STAT3 and its phosphorylation activities in DLBCL cells. This was consistent with the finding that phosphorylation is a pattern of activity regulation for STAT3 (39). In future studies, deletion of Bcl-2 and STAT3 using small interfering RNA will be performed to further reveal the mechanism of the anticancer effects of chidamide.

In conclusion, the present study demonstrated that chidamide can have a tumor-suppressive effect on DLBCL cells by inducing apoptotic cell death, which is regulated by the HDACs/STAT3/Bcl-2 pathway. As well as inhibiting the activity of HDAC, chidamide may also inhibit the activity of STAT3 to function as an apoptosis inducer in these DLBCL cells. Therefore, the present results suggested that chidamide has great potential as a therapeutic agent for the management of DLBCL. While these results are encouraging, the current research is limited to determine which of the ABC- and GCB-subtype cells or both are more sensitive to chidamide as only three cell lines (two ABC subtypes and one GCB subtype) were used. Additionally, the current data were only obtained from these cell lines, which possess their inherent limitations as any other in vitro models (40). Consequently, further research, including sufficient numbers of two subtypes of DLBCL cells and in vivo studies, is necessary to validate the antitumor effect of chidamide and its precise underlying cellular mechanism for its true clinical value to be revealed.

\section{Acknowledgements}

Not applicable.

\section{Funding}

This work was supported by Nature Science Project of Shanxi, China (grant nos. 201701D121165 and 201901D111190), the Research Project Supported by Shanxi Scholarship Council of China (gran no. 2020-194), Key R \& D Projects in Shanxi, China (grant no. 201703D421023), the Open Fund from Key Laboratory of Cellular Physiology (Shanxi Medical University), Ministry of Education, China (grant no. KLMEC/SXMU-202011) and the Shanxi '1331 Project' Key Subjects Construction, China (grant no. 1331KSC).

\section{Availability of data and materials}

The datasets used and/or analyzed during the current study are available from the corresponding author on reasonable request.

\section{Authors' contributions}

$\mathrm{BYu}$ and $\mathrm{LS}$ designed the project. $\mathrm{HZ}, \mathrm{FC}, \mathrm{KQ}, \mathrm{XM}$ and LW performed the experiments. HZ, BYa, YW, MB and ZL analyzed the data. HZ and BYa wrote the manuscript. All authors read and approved the final manuscript. BYu and LS confirm the authenticity of all the raw data.

\section{Ethics approval and consent to participate}

Not applicable.

\section{Patient consent for publication}

Not applicable.

\section{Competing interests}

The authors declare that they have no competing interests.

\section{References}

1. Kubuschok B, Held G and Pfreundschuh M: Management of diffuse large B-cell lymphoma (DLBCL). Cancer Treat Res 165: 271-288, 2015.

2. Li S, Young KH and Medeiros LJ: Diffuse large B-cell lymphoma. Pathology 50: 74-87, 2018.

3. Kong Y, Chen G, Xu Z, Yang G, Li B, Wu X, Xiao W, Xie B, Hu L, Sun $\mathrm{X}$, et al: Pterostilbene induces apoptosis and cell cycle arrest in diffuse large B-cell lymphoma cells. Sci Rep 6: 37417, 2016.

4. Hunt KE and Reichard KK: Diffuse large B-cell lymphoma. Arch Pathol Lab Med 132: 118-124, 2008.

5. Sehn LH and Gascoyne RD: Diffuse large B-cell lymphoma: Optimizing outcome in the context of clinical and biologic heterogeneity. Blood 125: 22-32, 2015.

6. Mondello P and Mian M: Frontline treatment of diffuse large B-cell lymphoma: Beyond R-CHOP. Hematol Oncol 37: 333-344, 2019.

7. Pan YR, Chen CC, Chan YT, Wang HJ, Chien FT, Chen YL, Liu JL and Yang MH: STAT3-coordinated migration facilitates the dissemination of diffuse large B-cell lymphomas. Nat Commun 9: 3696, 2018.

8. Tamma R, Ingravallo G, Albano F, Gaudio F, Annese T, Ruggieri S, Lorusso L, Errede M, Maiorano E, Specchia G and Ribatti D: STAT-3 RNA scope determination in human diffuse large B-cell lymphoma. Transl Oncol 12: 545-549, 2019.

9. Lu K, Li B, Zhang H, Xu Z, Song D, Gao L, Sun H, Li L, Wang Y, Feng $\mathrm{Q}$, et al: A novel silicone derivative of natural osalmid (DCZ0858) induces apoptosis and cell cycle arrest in diffuse large B-cell lymphoma via the JAK2/STAT3 pathway. Signal Transduct Target Ther 5: 31, 2020.

10. Ding BB, Yu JJ, Yu RY, Mendez LM, Shaknovich R, Zhang Y, Cattoretti $\mathrm{G}$ and $\mathrm{Ye} \mathrm{BH}$ : Constitutively activated STAT3 promotes cell proliferation and survival in the activated B-cell subtype of diffuse large B-cell lymphomas. Blood 111: 1515-1523, 2008.

11. Moskowitz AJ and Horwitz SM: Targeting histone deacetylases in T-cell lymphoma. Leuk Lymphoma 58: 1306-1319, 2017.

12. Bai X, Jiang H, Han G and He Q: Chidamide suppresses the glycolysis of triple negative breast cancer cells partially by targeting the miR33a5pLDHA axis. Mol Med Rep 20: 1857-1865, 2019.

13. Sun Y, Li J, Xu Z, Xu J, Shi M and Liu P: Chidamide, a novel histone deacetylase inhibitor, inhibits multiple myeloma cells proliferation through succinate dehydrogenase subunit A. Am J Cancer Res 9: 574-584, 2019. 
14. Wu YF, Ou CC, Chien PJ, Chang HY, Ko JL and Wang BY: Chidamide-induced ROS accumulation and miR-129-3p-dependent cell cycle arrest in non-small lung cancer cells. Phytomedicine 56: 94-102, 2019.

15. Ubink I, Bolhaqueiro ACF, Elias SG, Raats DAE, Constantinides A Peters NA, Wassenaar ECE, de Hingh IHJT, Rovers KP, van Grevenstein WMU, et al: Organoids from colorectal peritoneal metastases as a platform for improving hyperthermic intraperitoneal chemotherapy. Br J Surg 106: 1404-1414, 2019.

16. Livak KJ and Schmittgen TD: Analysis of relative gene expression data using real-time quantitative PCR and the 2(-Delta Delta C(T)) method. Methods 25: 402-408, 2001.

17. Best S, Hashiguchi T, Kittai A, Bruss N, Paiva C, Okada C, Liu T, Berger A and Danilov AV: Targeting ubiquitin-activating enzyme induces ER stress-mediated apoptosis in B-cell lymphoma cells. Blood Adv 3: 51-62, 2019.

18. Jin CY, Moon DO, Choi YH, Lee JD and Kim GY: Bcl-2 and caspase-3 are major regulators in Agaricus blazei-induced human leukemic U937 cell apoptosis through dephoshorylation of Akt. Biol Pharm Bull 30: 1432-1437, 2007.

19. Ning ZQ, LiZB, Newman MJ, Shan S, Wang XH, Pan DS, Zhang J, Dong M, Du X and Lu XP: Chidamide (CS055/HBI-8000): A new histone deacetylase inhibitor of the benzamide class with antitumor activity and the ability to enhance immune cell-mediated tumor cell cytotoxicity. Cancer Chemother Pharmacol 69: 901-909, 2012

20. Li Y and Seto E: HDACs and HDAC inhibitors in cancer development and therapy. Cold Spring Harb Perspect Med 6: a026831, 2016.

21. Williams SA, Chen LF, Kwon H, Ruiz-Jarabo CM, Verdin E and Greene WC: NF-kappaB p50 promotes HIV latency through HDAC recruitment and repression of transcriptional initiation. EMBO J 25: 139-149, 2006.

22. Lee SH, Yoo C, Im S, Jung JH, Choi HJ and Yoo J: Expression of histone deacetylases in diffuse large B-cell lymphoma and its clinical significance. Int J Med Sci 11: 994-1000, 2014.

23. Gloghini A, Buglio D, Khaskhely NM, Georgakis G Orlowski RZ, Neelapu SS, Carbone A and Younes A: Expression of histone deacetylases in lymphoma: Implication for the development of selective inhibitors. Br J Haematol 147: $515-525,2009$.

24. Wang M, Fang X and Wang X: Emerging role of histone deacetylase inhibitors in the treatment of diffuse large B-cell lymphoma. Leuk Lymphoma 61: 763-775, 2020.

25. Gao S, Li X, Zang J, Xu W and Zhang Y: Preclinical and clinical studies of chidamide (CS055/HBI-8000), an orally available subtype-selective HDAC inhibitor for cancer therapy. Anticancer Agents Med Chem 17: 802-812, 2017.

26. Chan TS, Tse E and Kwong YL: Chidamide in the treatment of peripheral T-cell lymphoma. Onco Targets Therapy 10: 347-352, 2017.

27. Li Q,Huang J, Ou Y,Li Y and Wu Y: Progressive diffuse large B-cell lymphoma with TP53 gene mutation treated with chidamide-based chemotherapy. Immunotherapy 11: 265-272, 2019.
28. Miao Y, Medeiros LJ, Li Y, Li J and Young KH: Genetic alterations and their clinical implications in DLBCL. Nat Rev Clin Oncol 16: 634-652, 2019.

29. Ngo VN, Young RM, Schmitz R, Jhavar S, Xiao W, Lim KH, Kohlhammer H, Xu W, Yang Y, Zhao H, et al: Oncogenically active MYD88 mutations in human lymphoma. Nature 470: $115-119,2011$

30. Compagno M, Lim WK, Grunn A, Nandula SV, Brahmachary M, Shen Q, Bertoni F, Ponzoni M, Scandurra M, Califano A, et al: Mutations of multiple genes cause deregulation of NF-kappaB in diffuse large B-cell lymphoma. Nature 459: 717-721, 2009.

31. Guan XW, Wang HQ, Ban WW, Chang Z, Chen HZ, Jia L and Liu FT: Novel HDAC inhibitor Chidamide synergizes with Rituximab to inhibit diffuse large B-cell lymphoma tumour growth by upregulating CD20. Cell Death Dis 11: 20, 2020.

32. Teng Y, Ross JL and Cowell JK: The involvement of JAK-STAT3 in cell motility, invasion, and metastasis. JAKSTAT 3: e28086, 2014.

33. Sgrignani J, Garofalo M, Matkovic M, Merulla J, Catapano CV and Cavalli A: Structural biology of STAT3 and its implications for anticancer therapies development. Int J Mol Sci 19: 1591, 2018.

34. Gharibi T, Babaloo Z, Hosseini A, Abdollahpour-Alitappeh M, Hashemi V, Marofi F, Nejati K and Baradaran B: Targeting STAT3 in cancer and autoimmune diseases. Eur J Pharmacol 878: 173107,2020

35. Mohassab AM, Hassan HA, Abdelhamid D and Abdel-Aziz M: STAT3 transcription factor as target for anti-cancer therapy. Pharmacol Rep 72: 1101-1124, 2020.

36. Ni L, Wang L, Yao C, Ni Z, Liu F, Gong C, Zhu X, Yan X, Watowich SS, Lee DA and Zhu S: The histone deacetylase inhibitor valproic acid inhibits NKG2D expression in natural killer cells through suppression of STAT3 and HDAC3. Sci Rep 7: 45266, 2017.

37. Hoesel B and Schmid JA: The complexity of NF- $\kappa B$ signaling in inflammation and cancer. Mol Cancer 12: 86, 2013.

38. Lu L, Zhu F, Li Y, Kimpara S, Hoang NM, Pourdashti S and Rui L: Inhibition of the STAT3 target SGK1 sensitizes diffuse large B cell lymphoma cells to AKT inhibitors. Blood Cancer J 9: 43, 2019.

39. Belo Y, Mielko Z, Nudelman H, Afek A, Ben-David O, Shahar A, Zarivach R, Gordan R and Arbely E: Unexpected implications of STAT3 acetylation revealed by genetic encoding of acetyl-lysine. Biochim Biophys Acta Gen Subj 1863: 1343-1350, 2019.

40. Zhang R, Lyu C, Lu W, Pu Y, Jiang Y and Deng Q: Synergistic effect of programmed death-1 inhibitor and programmed death-1 ligand-1 inhibitor combined with chemotherapeutic drugs on DLBCL cell lines in vitro and in vivo. Am J Cancer Res 10: 2800-2812, 2020.

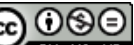

This work is licensed under a Creative Commons Attribution-NonCommercial-NoDerivatives 4.0 International (CC BY-NC-ND 4.0) License. 